\title{
Analyze of Frequency Selective Surfaces By Hybrid MOM-PO-GTD Method
}

samir mendil ( $\square$ myemail.contact@gmail.com )

Ecole Nationale d'Ingenieurs de Tunis https://orcid.org/0000-0003-2437-2493

Taoufik Aguili

Ecole Nationale d'Ingenieurs de Tarbes

Research

Keywords: frequency selective surfaces, general theory of diffraction (GTD), microwave frequency, spacial filters, periodic structures

Posted Date: December 31st, 2019

DOI: https://doi.org/10.21203/rs.2.19810/v1

License: (c) (1) This work is licensed under a Creative Commons Attribution 4.0 International License. Read Full License 


\title{
Analyze of Frequency Selective Surfaces By Hybrid MOM-PO-GTD Method
}

\author{
MENDIL SAMIR (myemail.contact@gmail.com) and AGUILI TAOUFIK(taoufik.aguili@gmail.co
}

December 27, 2019

\section{Abstract}

This article intends to analyze the Diffraction phenomena of the incoming wave and provide a new approach for analyzing the frequency selective surface (Fss) by using a hybrid method combining Moment Method (MoM), optical physics (PO) with General theory of Diffraction (GTD). the frequency selective surface (Fss) is a periodic surface with identical two-dimensional arrays of elements arranged on a substrate dielectric. An incoming plane wave will either be transmitted (bandwidth) or reflected (stopband), completely or partially, depending on the nature of the array element. Today, FSSs have been extensively studied and there is tremendous growth in its design and implementation for different applications at the microwave to optical frequency ranges. In this review article, we present a new hybrid method form on Moment method and GTD for analyzing different categories of FSS based on the design of the structure, the array elements used, and applications. We also focus on the effects of diffraction, methodology, experimental verifications of design examples, as well as on prospects and challenges, particularly in the microwave regime. We highlight their important performance metrics, especially about progress in this area could facilitate advanced electromagnetic innovation.

\section{Introduction}

The Frequency Selective Surface (FSS )mark their presence in many applications, including reflectors, antenna radomes, radar-absorbing materials, polarizers, and composite metamaterials. Since this area of research is relatively old but extremely fast-growing, it carries a wide range of applications and theories as many designs and analysis tools have

\footnotetext{
Keywords:

Frequency selective surfaces; General theory of diffraction (GTD);

microwave frequency; spatial filters; periodic structures;
} 
been developed. However, significant problems related to the theoretical and practical importance still have to be solved, and requires more work, for example, the development of double-curve FSS, non-periodic FSS.

According to the definition given in [1], FSS is metasurfaces that simply present an electrical response [2]. Since to adapt the frequency selectivity to the transmission/reflection characteristics, only the electric polarization can be sufficient. conforming to the theory of antenna and microwave engineering, these surfaces are made by planar and periodic networks of metal patches of different shapes. The patch has a negligible thickness compared to the wavelength, although it is large enough contrast with the depth of the skin of the metal. Both, metamaterials and metasurfaces (MSs) are fast-growing areas of research, with their use, spatially varying EM or optical responses can be obtained at will, with diffusion phase, amplitude, and polarization. Through a good selection of materials and design, the ultra-thin structure of MSs can suppress the detrimental and undesirable losses in the direction of wave propagation. When the considering polarization response, all metasurfaces can be classified based on the operating principle of a matrix element, i-e their functionalities (frequency selective surfaces (FSS), high impedance surfaces, perfect absorbents, reflective surfaces, etc.). Therefore, such a structure can perfectly be estimated as a tiny network of perfect conductive resonant elements. This approximation is applied to the complementary structures of the FSSs i-e aperture, with it faces a limitation when the surface of the aperture cell becomes equal to the elementary cell (mesh type). Square and hexagonal mesh cells have generally been used and are also referred to as a capacitive grid. The existence of the resonance size of the array element causes the emergence of sidelobes in the transmitted and reflected fields, which are the defining characteristic of FSS. However, compared to the FSS, the resonant element and the elementary cell of the metasurface are relatively much smaller than the wavelength and allow the elimination of grating lobes in the frequency response. Therefore, FSS in the terahertz domain is usually called metasurfaces [3]. in recent years, dispersion properties of FSS have been explored using approximate analytical techniques, which imply an equivalent circuit method (GEC) for analyzing the characteristics of the transmission line (quasi-static approximation). However, with the growth of more complex structures, advanced numerical methods have been introduced that use periodic boundary conditions (PBCs) allowing for a fairly simple design analysis. Some of them include the finite element method (FEM), the moment method (MoM), the finite-difference time-domain method (FDTD) and the integral equation method (boundary element) (IEM / BEM) [4-10]. A well-known technique is the IEM / BEM used in combination with MoM [1114]. Various designs of FSSs and schemes for examining their SE characteristics are well presented in [2].

Therefore, in free space, and for certain frequency range, an FSS is capable of transmitting or blocking EM waves; stying Diffraction phenomena of these EM waves and its interaction with the complex form of this structures explored more the properties of FSS and correct some parameters.

This paper targets to

-The link among their crucial theoretical, structure geometry and significant performance 
parameters due to Diffraction phenomena analyze;

- A joining numerical method with asymptotic one General Theory Of Diffraction (GTD) in the new approach of analyzing;

- Highlight the important of Diffraction phenomena on their performance.

\section{Study of Diffraction of Incident Wave in FSS Structure based on "metallic frame"}

In this section, we choose to study at the beginning a simple form "metallic frame" as FSS element. presented in fig.1

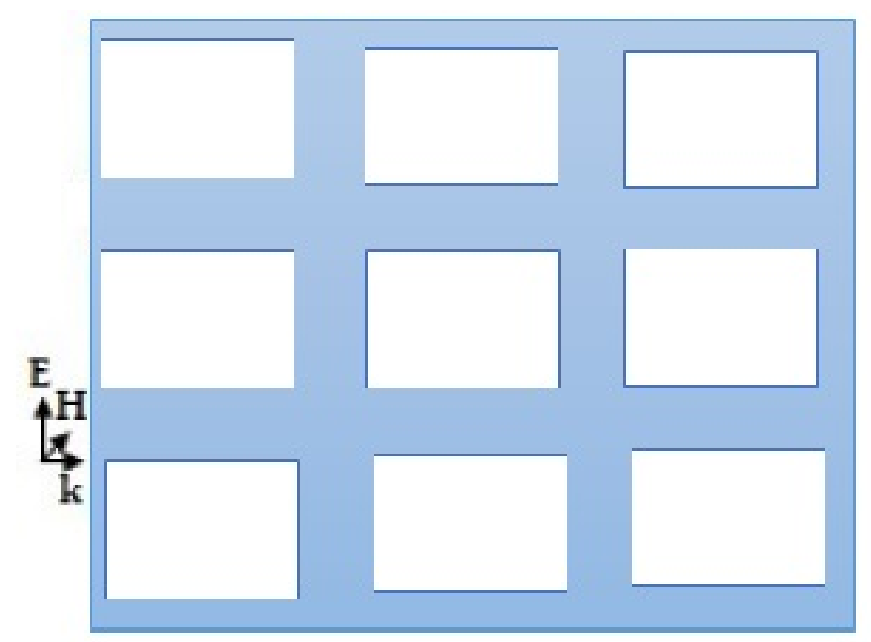

Figure 1: Surface waveguide made of metallic frames.

Periodic perfectly conducting surfaces of electricity (PEC) are the easiest to understand theoretically because they admit only sources of electrical current J. Frequency selective surfaces are frequently stratified in the normal direction to the plane of the surface. In other words, all dielectrics are stratified and all-metal conductors are also considered stratified, and they will be considered perfectly planar. As a result, we exclude metal (wires perpendicular to the FSS plane) that could potentially connect currents from different layers of the FSS structure. With this kind of stratified structure in mind, we can then use a plane wave extension for the fields inside and around the FSS, because plane waves are the eigenfunction of the equation.

we consider an infinite 2D periodic surface occupying the entire $\mathrm{x}-\mathrm{y}$ plane, and assume a discrete plane wave expansion for all currents, fields and potentials to solve,with, $l_{x}$ and $l_{y}$ dimension of the unit cell in (x-y), $k=\frac{2 \pi}{\lambda}, \lambda:$ wavelength, $\theta_{0}, \phi_{0}$ are the direction of an incident plane wave with the FSS regarded as lying in the $\mathrm{x}-\mathrm{y}$ plane.

Here we notice the singularity of the branch point in the equation above (the inverse singularity of the square root), which is not a problem thanks to the discrete spectrum, 
as long as the wavelength is not never equal to the spacing of the cells in our case lambda $<\left(D_{x}, D_{y}\right)$. With this, the boundary condition of the electric field at the surface of the PEC material in a unit cell becomes

$$
E^{i n c}(x, y)=E^{i n c}\left(\alpha_{0}, \beta_{0}\right) \exp ^{j\left(\alpha_{0} x+\beta_{0} y\right)}(1)
$$

Again, we limit our attention to the $\mathrm{x}, \mathrm{y}$ components of currents and fields, which lie in the plane of the diffuser. In the following, we begin to study a diffraction phenomenon for a single element of FSS, as presented in figure 2 .

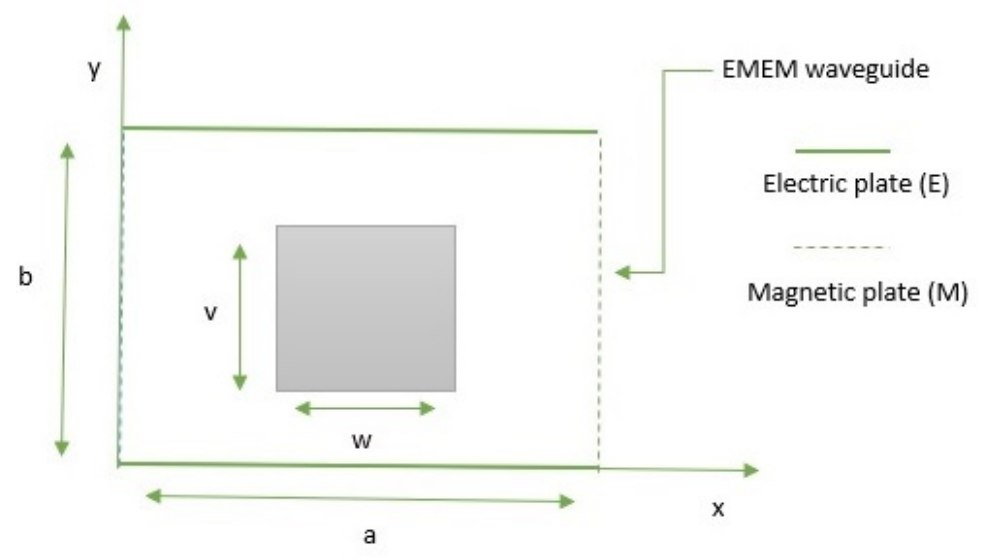

Figure 2: single Cell of FSS in waveguide.

its consist of a metal plate placed in a rectangular waveguide. this waveguide is infinite, lossless and symmetric through discontinuity planes. they are associated with (EMEM) boundaries: two perfect magnetic plates $(\mathrm{M})$ on the lateral sides and two perfect electric (E) on the top and bottom. the dimensions of the structures are : $a=$ $17 \mathrm{~mm}, \mathrm{~b}=7 \mathrm{~mm}, \mathrm{w}=2 \mathrm{~mm}$ et $\mathrm{v}=2 \mathrm{~mm}$.

\subsection{Application of the Numerical Method (MOM)}

The application of MoM method begins by writing the boundary conditions form an integral equation defined on the surface plate, which allows the reduction of the dimension problem. But since we increase the structures' complexity we complicate the problem more and more. Here, the use of equivalent circuits based on transposition fields simplifies the formulation of the integral equation and its treatment. The GEC method expresses the unknown electromagnetic boundary conditions, it represents the electric image of the studies structures for describing discontinuity and structured environment. The test functions model the virtual sources without storing energy in the plane of discontinuity. An impedance operator expressed this discontinuity environment represents boundary condition on each side of the discontinuity surface. the wave excites the discontinuity surface represented by real source with delivery energy. The GEC 
method extends the Kirchhoff's laws used in (I, V) to the (H, E) concept in Maxwell's conformity. the GEC for the studies structures are modeling by figure 3

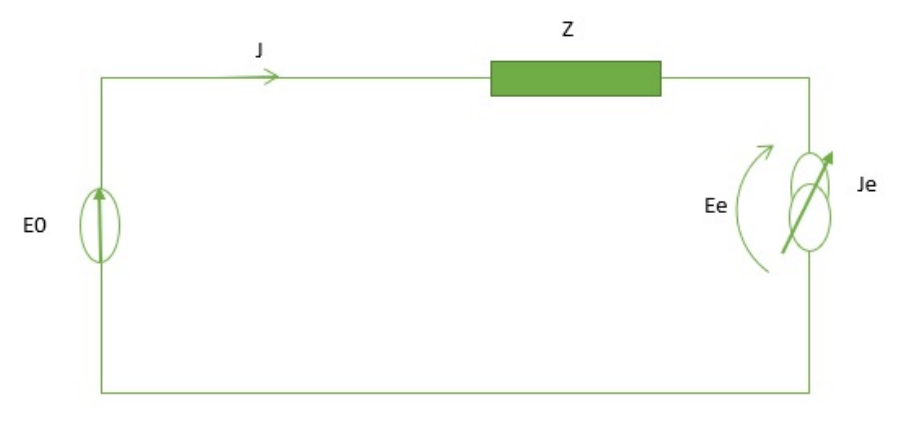

Figure 3: the Equivalent Circuit Method

with the Impedance operator $\mathrm{Z}$ defined as:

$$
Z=\sum_{m}\left|F_{m}>z_{m}<F_{m}\right|(2)
$$

And $J_{e}$ virtual source defined on the metallic domain as :

$$
J_{e}=\sum x_{p} g_{p}(3)
$$

with $x_{p}, p \in\left(1,2,3, \ldots, N_{e}\right)$ unknown coefficients then we obtain the equation system :

$$
\left\{\begin{array}{l}
J=-J_{e} \\
E_{e}=E_{0}+Z J_{e}
\end{array}\right.
$$

The surface current $\mathrm{J}$ expressed in modal basis function $f_{m}, m \in(1,2 \ldots, M)$ weighted by unknown coefficients $I_{m}, m \in(1.2, \ldots, M)$

$$
J=\sum I_{m} f_{m}(5)
$$

After the application the Galerkin's method and Kirchhoff's laws we obtain the following equation system :

$$
\left\{\begin{array}{l}
I=-A^{T} X \\
0=A V_{0}+B X
\end{array}\right.
$$

After resolves the previous system, we obtain the input impedance :

$$
Z_{\text {in }}=\frac{1}{A^{T} B^{-1} A}(7)
$$

We can also now evaluate the weighting coefficients $x_{p}$, surface current $\mathrm{J}$ and diffracted field. The studies structure is formed by metallic and dielectric patterns $S=S_{M}+S_{D}$, 
Structured on current and field proprieties on S, $S_{M}$ is the metallic sub-domain where the field is null and it's dual current not null. In other parts, $S_{D}$ is the dielectric subdomain where the current is null and the field is not null. The concept of a secondary source is to put together all field and current representations in an only one which will be valid in all point of the domain $\mathrm{D}$, for our problem, $J_{e}$ is the virtual source of current defined on metallic domain and defined as set of known test functions weighted by unknown coefficients. At this point now and according to the MOM method we must choose a set of sinusoidal or triangular test functions a wrong or not accurate choice can complicate the problem. In the following, we introduce a new test function which converges quickly and replaces the set of lot sinusoidal test function.in the first step we use the PO to find the current on the metal and the next step we use the GTD to find the current on the edges and tips.

\subsection{Methods: Hybrid Approach MOM-PO-GTD}

\subsubsection{Calcul of Optical Physics $\left(J_{P O}\right)$ Current}

We propose to determine the first step of the new test function by the optical physics method. This approach is based on the determination of the current flowing through the metal when it is illuminated by an electromagnetic field as we see in figure 4 .

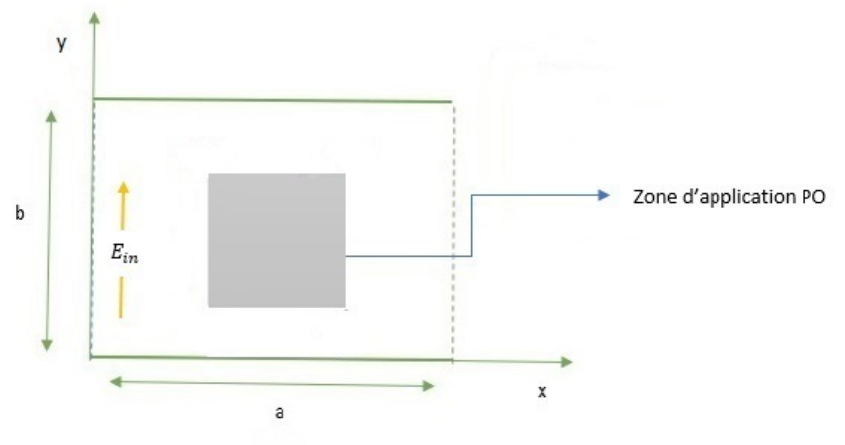

Figure 4: Mechanism of Optical Physics on the FSS unit

$E_{i n c}$ and $H_{i n c}$ electric and magnetic fields associated with the fundamental mode of the waveguide (TEM). Like the surface of an object illuminated by an incident magnetic field $\vec{H}_{i} n$, an electric current density called $\vec{J}_{p o}$ found in this surface this current in related to the magnetic field by the following equation.

$$
\vec{J}=2 \vec{H}_{i n} \wedge \vec{z}(8)
$$

with $\vec{H}_{\text {in }}$ the incident magnetic field on the waveguide. $\vec{y}$ the unit normal vector. The $\vec{H}_{i n}$ related to the incident electric field by :

$$
\operatorname{rot}\left(\overrightarrow{E_{i n c}}\right)=-i \omega \mu_{0} \vec{H}_{i n}(9)
$$


the current is evaluate as :

$$
\vec{J}_{p o}=\frac{2 \beta}{\omega \mu_{0}} \sqrt{\frac{1}{a}} \vec{y}(10)
$$

$\beta$ is the waveguide constant of propagation, $\omega$ the wave pulsation and $\mu_{0}$ the permeability of the air.

\subsubsection{Calcul of General diffraction theory Current $\left(J_{G T D}\right)$}

Diffraction it's considered as a local phenomenon at very high frequencies, the GTD relies on known solutions of diffraction such as diffraction by half-plane, dihedral, cone, cylinder. From these solutions in the far-field, we propose to build an asymptotic evaluation of $J_{G T D}$ that converge better to the exact solution.

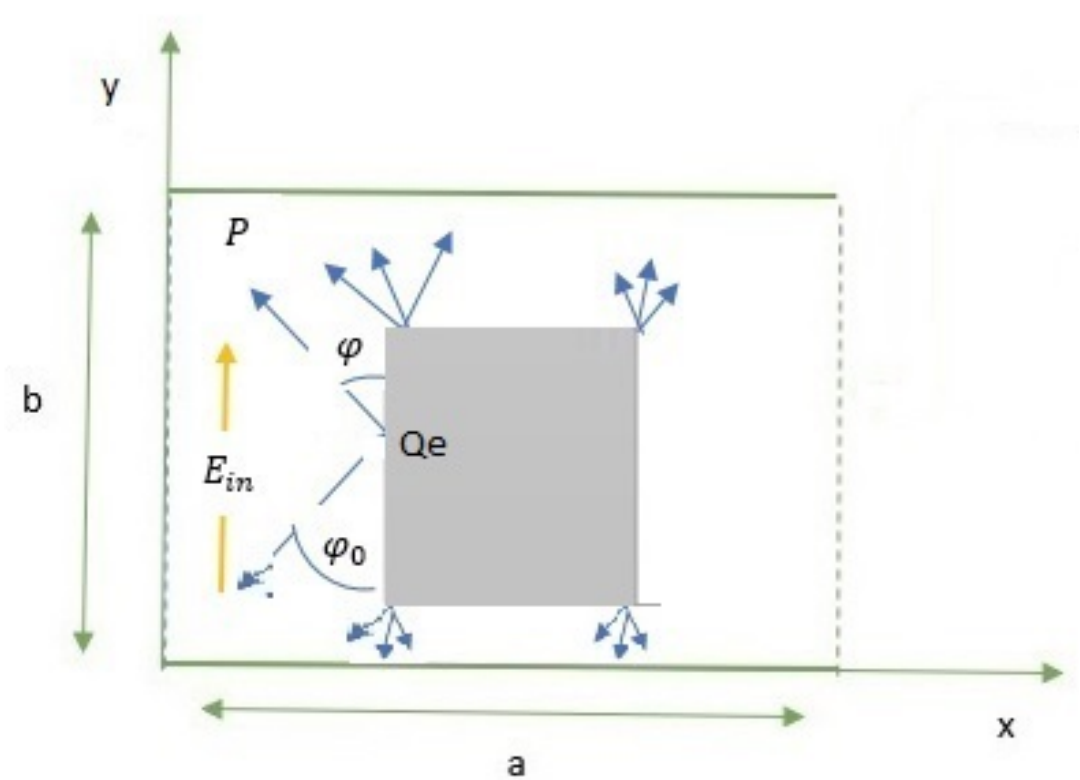

Figure 5: Diffraction of incident wave on the Edge and tips of the FSS unit

In the figure 5, a plane wave at normal incidence at the point $\mathrm{P}$ can be expressed in TEM fondamendal Mode as :

$$
E^{i}=\frac{1}{\sqrt{a}} \exp ^{-j k z}(11)
$$

The electromagnetic plane wave incident on the perfectly-conducting wedge, the total electric field intensity $\mathrm{E}$ and the total magnetic field intensity $\mathrm{H}$ in the region surrounding the wedge composed into a total of transverse and axial (to $\hat{x}$ )

Refered to P.H Pathak and R.G kouyoumjian work, the field diffracted by this edge will be: 


$$
E_{y}^{d}=E_{y}^{i} \sqrt{\sin \phi_{0}} \cdot D \frac{\exp ^{-j k_{t} \rho}}{\sqrt{\rho}} \exp ^{-j k_{z} z}(12)
$$

and

$$
H_{x}^{d}=H_{x}^{i} \sqrt{\sin \left(\phi_{0}\right)} D \frac{\exp ^{-j k_{t} \rho}}{\sqrt{\rho}} \exp ^{-j k_{z} z}(13)
$$

where $E_{y}^{i}$ and $H_{x}^{i}$ are evaluated at $Q_{E}$, the point of incidence on the edge, we must calculate the following Coeifficent:

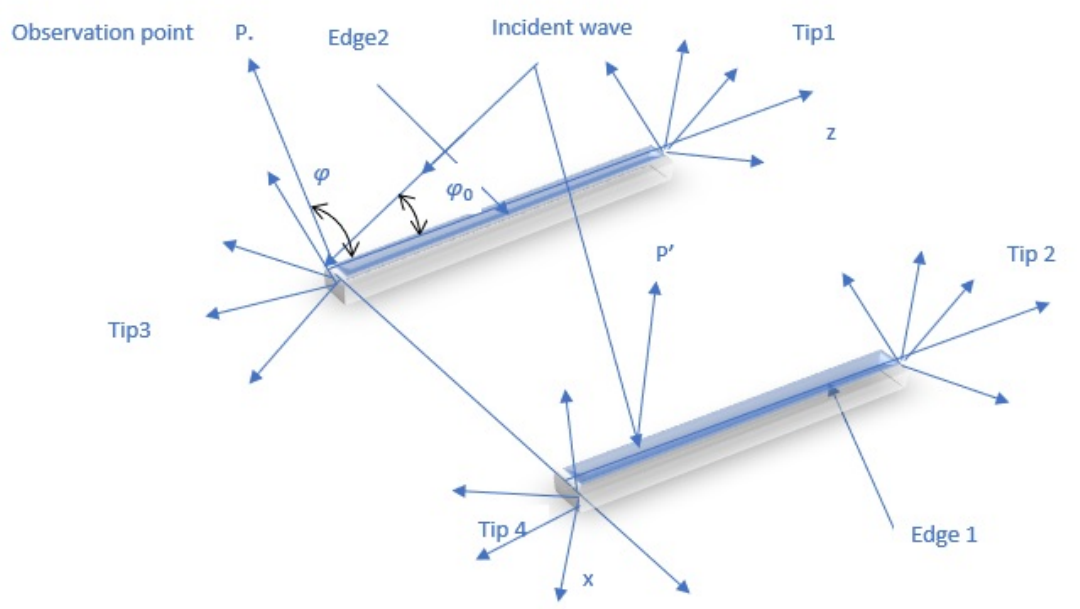

Figure 6: Modelization of FSS unit Edge and tips

From figure 6 , the fine metal wire with $\mathrm{b}$ radius and 1 length we introduce their diffractions coefficients.

For thin edge:

$$
D_{\text {edge }}=\exp ^{i \frac{\pi}{4}}\left(\frac{\pi}{2 k \sin ^{2} \phi_{0}}\right)^{\frac{1}{2}} \frac{1}{\left(\gamma+\log \left(\frac{k b \sin \phi_{0}}{2 j}\right)\right)}(14)
$$

with $\gamma=0.577$ is the Euler constant. and $\phi_{0}$ is angle between tangent to the edge and incident wave, $\mathrm{b}$ and $\mathrm{l}$ is respectively the radius and the length of this fine edge, we assume in the follows that the product of wire radius $\mathrm{b}$ and the wave number $\mathrm{k}$ is small and we are justified in neglecting the terms $O\left((k b)^{2}\right) \ln n(k b)$.

For the tips :

$$
D_{\text {tip }}=\frac{1}{k} \frac{i}{2 \ln \frac{2 j}{\gamma k b \sin \phi_{0}} \ln \frac{2 i}{\gamma k b \sin \phi}} \ln . n \cdot\left(\frac{i}{\gamma k b \sin \left(\frac{\phi_{0}}{2}\right) \sin \left(\frac{\phi}{2}\right)}\right)-\frac{\operatorname{tg}\left(\frac{\phi_{0}}{2}\right) \operatorname{tg}\left(\frac{\phi}{2}\right)}{\cos (\phi)+\cos \left(\phi_{0}\right)}(15)
$$


with $\mathrm{n}=3$.

finally the difraction coeificient is defined as :

$$
\bar{D}=2 D_{\text {edge }}+4 D_{\text {tips }}(16)
$$

the total diffracted field arround the structure is expressed as :

$$
E_{d}^{t}=E_{\text {edge } 1}^{d}+E_{\text {edge } 2}^{d}+E_{t i p 1}^{d}+E_{t i p 2}^{d}+E_{t i p 3}^{d}+E_{t i p 4}^{d}(17)
$$

Or

$$
\operatorname{rot}\left(E_{d}^{t}\right)=-i \omega \mu_{0} \vec{H}_{d}(18)
$$

then,

$$
J_{g t d}=\left(\vec{H}_{d}+\vec{H}_{i}\right) \wedge \vec{z}(19)
$$

The GTD current is concluded by the followillg expression:

$$
\overrightarrow{J_{\text {gtd }}}=\frac{\beta}{\omega \mu_{0}}\left[\frac{1}{\sqrt{a}}\left(2 D_{\text {edge }}+4 D_{\text {tip }}\right)+2 \sqrt{\frac{1}{a}}\right] \vec{y}(20)
$$

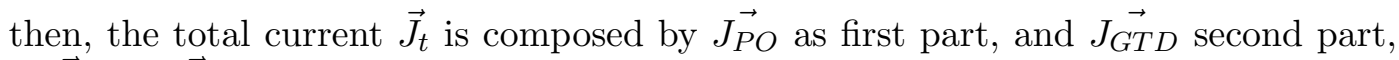
$\overrightarrow{J_{t}}=\overrightarrow{J_{P O}}+\overrightarrow{J_{G T D}}$ and it will be used as a single test function in our hybrid method.

$$
\vec{J}_{t}=\left[\left(\frac{\beta}{\omega \mu_{0}}\left[\frac{1}{\sqrt{a}}\left(2 D_{\text {edge }}+4 D_{\text {tip }}\right)+2 \sqrt{\frac{1}{a}}\right]\right)+\frac{2 \beta}{\omega \mu_{0}} \sqrt{\frac{1}{a}}\right] \vec{y}(21)
$$

As we see we notice the appearance of the coefficients of diffractions in the expression of the current $J_{t}$, therefore, we highlight, the effect of the geometry (edge, tips, etc.) of the structure of study. According to the Galerkin's method, to determinate, a structure's input impedance and distributions of field and current, several projections between test and basis function $\left(g_{p}, f_{m}\right)$ must be achieved.

Considering our approach, the single test function is divided into two parts ( $J t=$ $\left.J_{p o}+J_{g t d}\right)$, its projection on the waveguide's basis functions lead to obtaining the two integrals in the following:

$$
\begin{gathered}
\left\langle J_{t}, f_{m}\right\rangle=\left\langle J_{p o}, f_{m}\right\rangle+\left\langle J_{g t d}, f_{m}\right\rangle(22) \\
\left\langle J_{t}, f_{m}\right\rangle=\int_{x_{1}}^{x_{2}} \int_{y_{1}}^{y_{2}} f_{m} J_{p o} d x d y+\int_{x_{1}}^{x_{2}} \int_{y_{1}}^{y_{2}} f_{m} J_{g t d} d x d y(23)
\end{gathered}
$$

For $J_{g t d}$ Current, the width of the edge is very small, assimilated to a wire antenna, then we have a variation according to $y$

$$
\left\langle J_{g t d}, f_{m}\right\rangle=\int_{y_{1}}^{y_{2}} f_{m} J_{g t d} d y(24)
$$


To simplify the calculation of the equation (31), we will solve it numerically using the rectangle method (midpoint. method) expressed as :

$$
\int_{y 1}^{y_{2}} f(y) d y=\sum_{i=1}^{n} f\left(y_{i}\right) d y(25)
$$

We've studied up to now only one element of the FSS structure, to have the whole, and we consider that all unit spacing by $l_{x}$ et $l_{y}$ great than wavelength to eliminate the effect coupling in FSS structure, we obtain

$$
\sum_{j}\left[\sum_{m n} \frac{J_{t}\left(-\alpha_{m},-\beta_{n}\right) G_{m n} g_{j}\left(\alpha_{m}, \beta_{n}\right)}{\sqrt{k^{2}-\alpha_{m}^{2}-\beta_{n}^{2}}}\right]=-J_{t}\left(-\alpha_{0},-\beta_{0}\right) \cdot E^{i n c}\left(\alpha_{0}, \beta_{0}\right)(26)
$$

\section{Numerical Results and Discussion}

\subsection{Convergence study}

In our problem, we use a single test function, so the convergence study is done as a function of Number of modal basis functions. we present in the fig. 7 the input impedance $Z_{i n}$ of the considered structures against the number of modes of waveguides in the frequency range $[10 \mathrm{Ghz}, 12 \mathrm{Ghz}]$. It is clear that for all structures, the $Z_{\text {in }}$ 's convergence is obtained for 100 modes of waveguides. We note that the convergence is rapidly reached compared to cases using sinusoidal or triangular test functions. This conforms wiith the law $\left(\frac{P}{N}<\frac{\text { Metalsurface }}{\text { totalsurface }}\right)$ in a domain of discontinuity, whith $\mathrm{P}$ is test functions Number and $\mathrm{N}$ is basis functions Number.

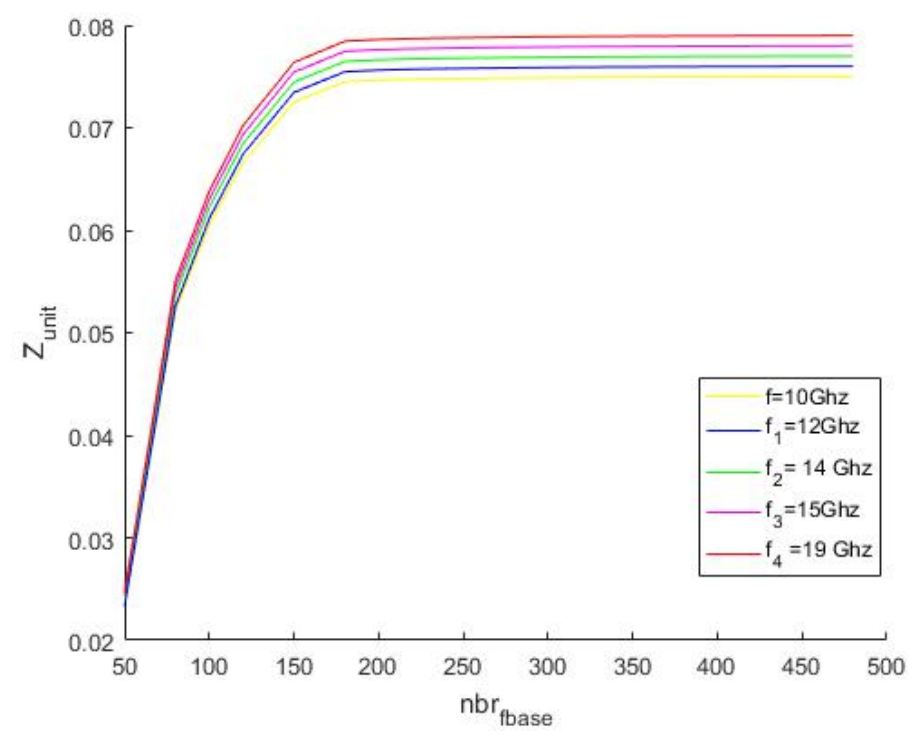

Figure 7: Input Impedance for different Number of Basics Function 


\subsection{Gain and Comparison between (MoM) Method and Hybrid method}

To evaluate our approach, we will compare for FSS structures, the results provided by the method of the moments using the functions of the sinusoidal tests, for the input impedance and the distribution of the current with those found by the hybrid method.

At Begining, the imaginary part of input impedance is computed for the frequency range $[10 \mathrm{Ghz}, 12 \mathrm{Ghz}]$ and drawn in figure 8 .

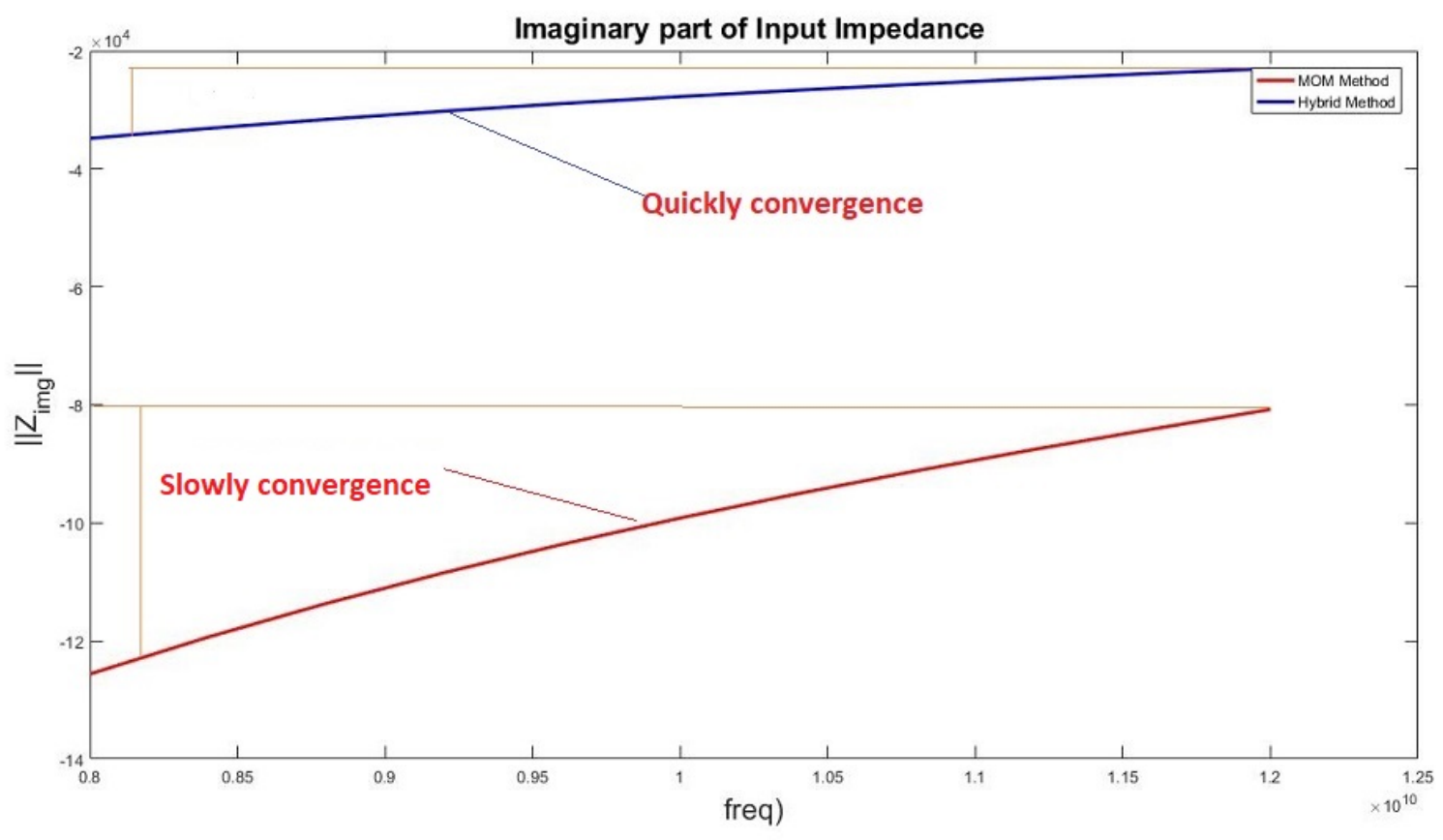

Figure 8: Comparaison between MoM Method and Hybrid Method of Imaginary Part of Input Impedance on the FSS Element

to highlight the importance of the geometric shape withdraw in fig 8 the imaginary part of input impedance for hybrid method, and we demonstrate here, that the hybrid method converges quickly than others. For the structure, the obtained results by the two methods are in agreement for the frequency in the previous range. We reporting here, that the numbers of sinusoidal test and basis functions used for MoM method are respectively 40 and 3000 for the structure, whereas our approach consists in using only one hybrid test function with 100 basis functions. Another very important remark is that the speed of the convergence of our hybrid approach is very fast compared by the moment's method its clear in the slopes of the two curves fig7a or fig 8, caused essentially by the diffraction coefficients used. The convergence is achieved quickly since the number of test and basis functions is decreased the number of operation is reduced also. 


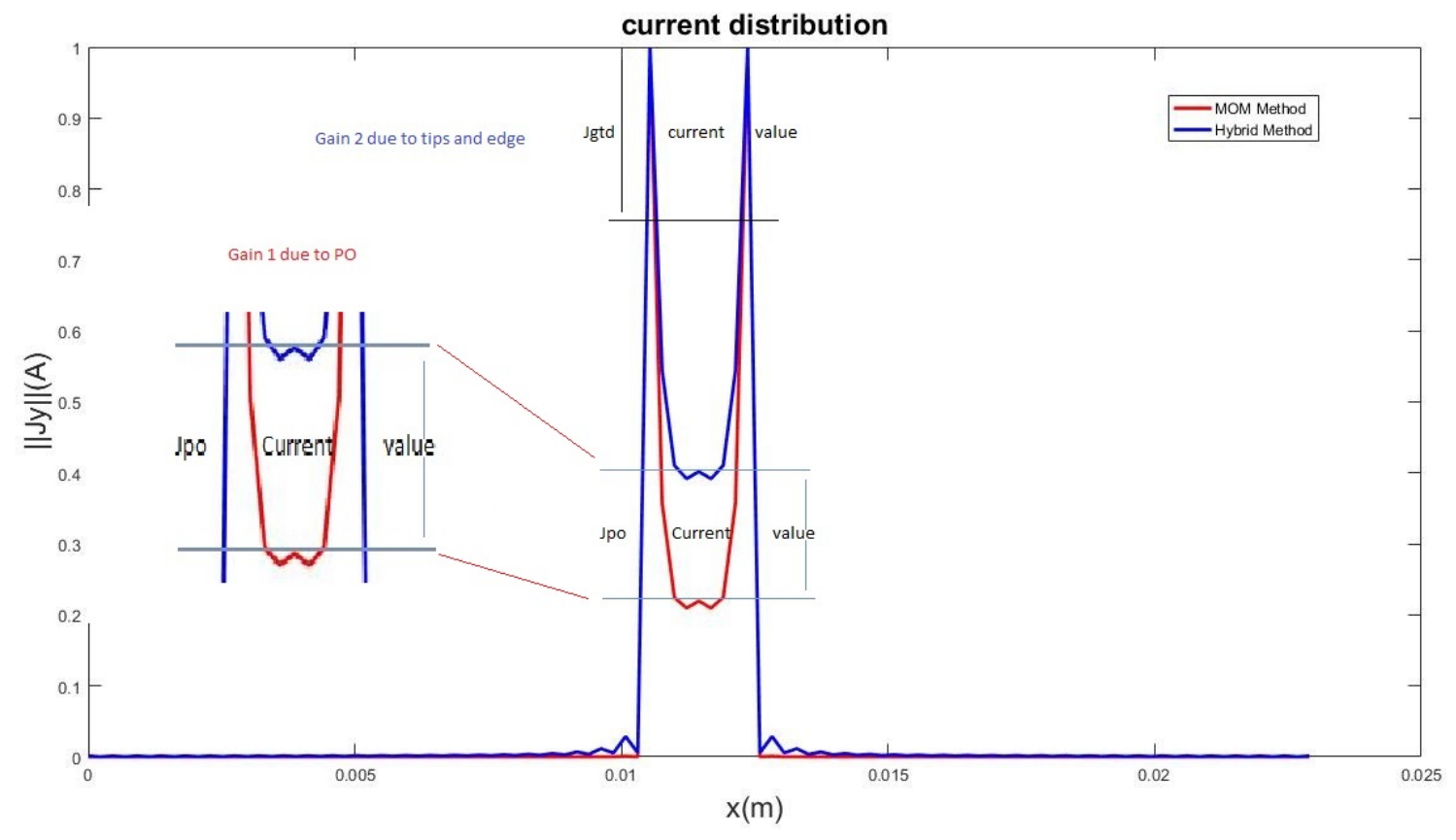

Figure 9: Comparaison between MoM Method and Hybrid Method of Current Distribution on the FSS Element

The Hybrid current distribution is given in fig 9 and its compared to those obtained with MoM method. A good agreement between the two methods is assured.it is also clear that the current on edges, approximated by the asymptotic method GTD using the diffraction coefficient, will satisfy boundary conditions: it's maximum on the edge of each FSS element. This demonstrates the development of the proposed test function. 


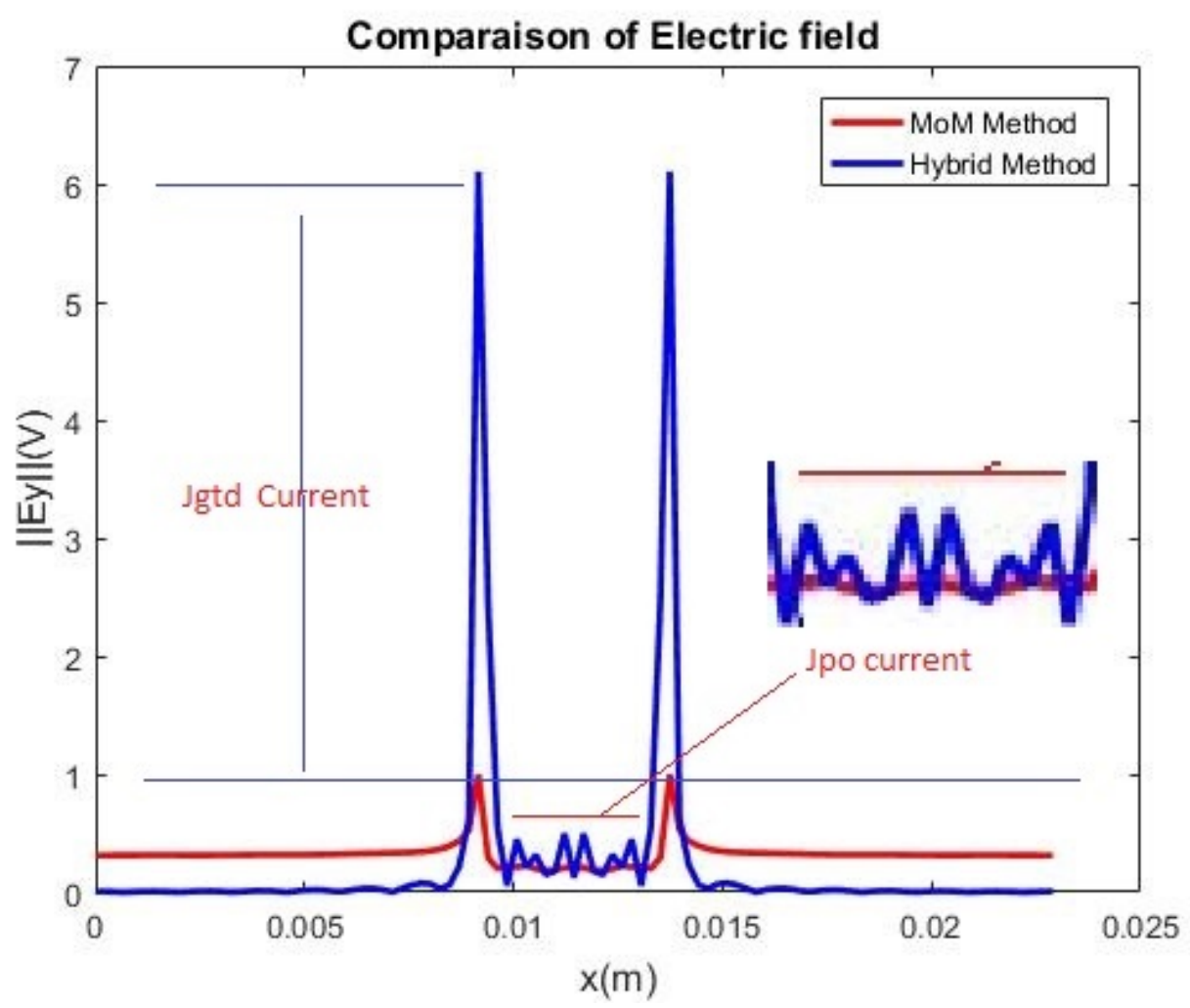

Figure 10: Comparaison between MoM Method and Hybrid Method of Electric Field on the FSS Element

Similarly, fig.10 shows the distribution of the electric field. Like input impedances and current, there is perfect accordance between the two methods with verification of boundary conditions. In further, the intensity of the electric field is very hight in hybrid method, caused essentially by the secondary source (tips, edge) that it's accounting in our approach, and negligent in Moment method. the number of basis functions is reduced too ( no more calculate operation to do), and good accuracy of the new hybrid approach is proved. Let's note that this hybrid method, the intent of this paper, requires less numerical complexity and needs less storage than the MoM method, which makes its use more practical and evidencing the effect of the geometric form. also by using a single test function, the size of the matrix becomes $1 \times 1$ instead of $30 \times 30$ in case of sinusoidal test functions. the fig 11 and 12 shown the distribution current on the fss's structure on $\mathrm{X}$-axes fig 11 and $\mathrm{Y}$-axis fig 12, for the moment method and hybrid method. in Y-axis, the draw of hybrid method overlaps it's of the moment method. 


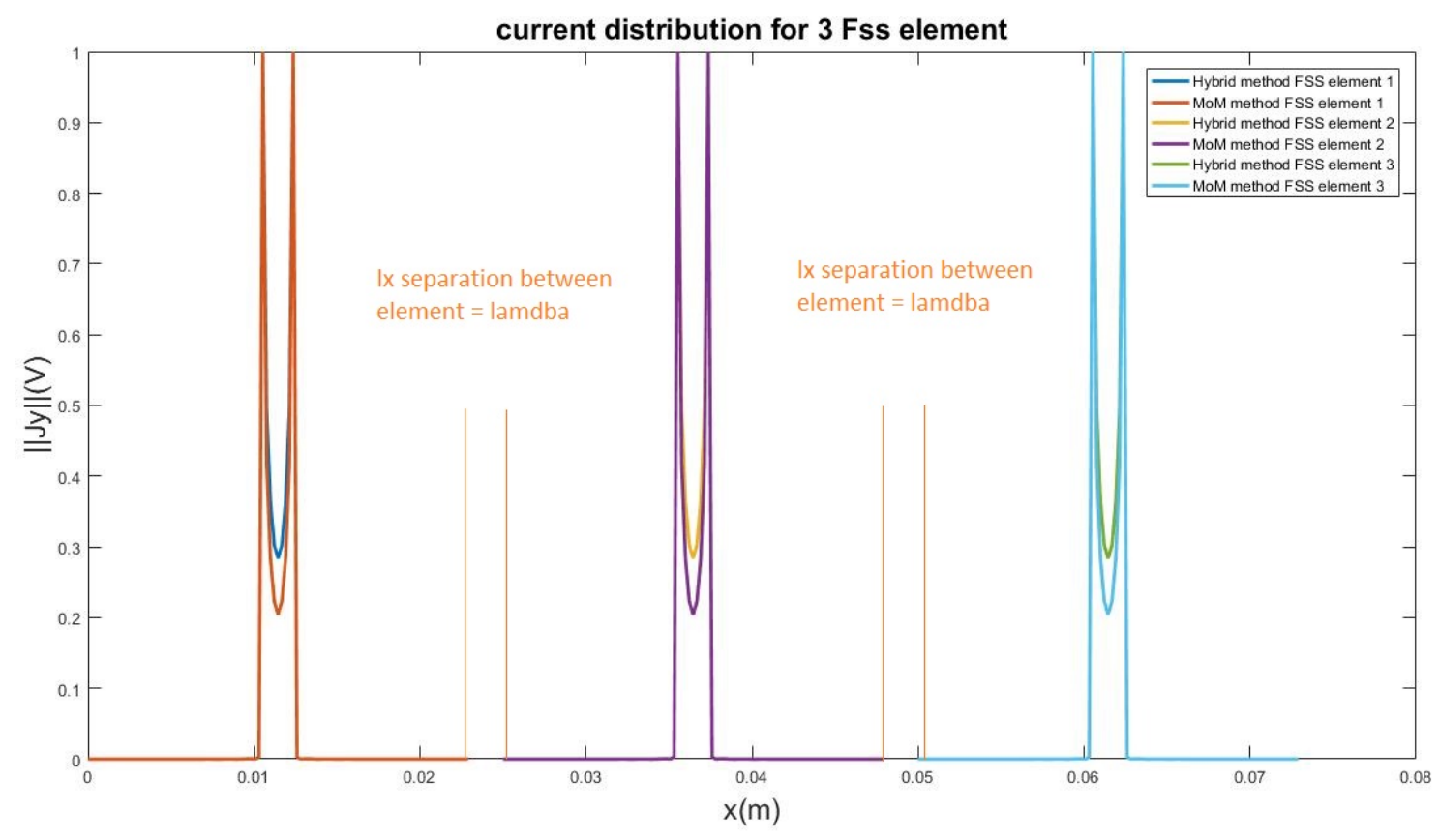

Figure 11: Current Distribution on the three FSS Element on X-axis

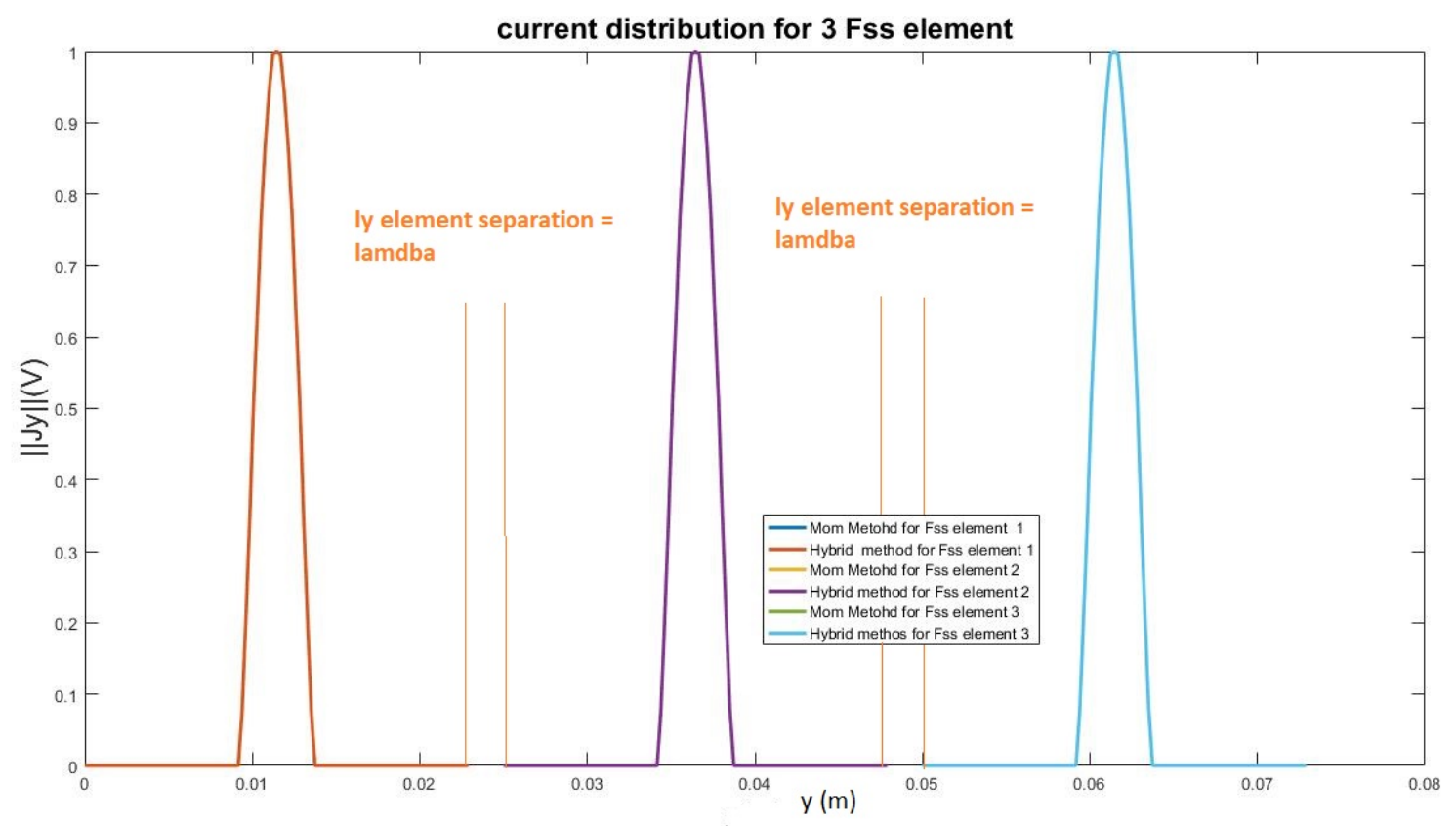

Figure 12: Current Distribution on the three FSS Element on Y-axis 
In the following, we present the gain that our new approach, gives in term of convergence and time calculation against the reference (MoM) method. Table1 and Table 2 summarizing the benefits of our approach. Table1 represents the number of tests and modal basis functions needed to obtain the convergence of input impedance for the rang $[8 \mathrm{Ghz}, 12 \mathrm{Ghz}]$, (shown in figure 6) and gives also the required time needed, for two values of Diffraction Coefficient D1 and D2 for different angle of observation $\phi_{1}=\frac{\pi}{4}$ respectively, $\phi_{2}=\frac{\pi}{6}$.in fact to calculate the input impedance we need $16.78 \mathrm{~s}$ using our hybrid method, respectively $1431.569 \mathrm{~s}$ using MoM Method with sinusoidal test function. further, by using our approach for the analyzed structure, only 100 waveguide's modes are required to obtain the convergence. respectively, 3000 waveguides modes and 16 test function for Mom Method to obtain the convergence. hence, Table 2 gives the time in seconds of calculation and the number of tests and basic function needed to get the convergence of the study structure when evaluating the current and electric fields. we accomplish the comparison between the MoM and the hybrid approach.

Table1. Comparison of computing time consumed between the MoM and hybrid approach to computing $Z_{i n}$ input impedance in the range [8-12Ghz] for A unit Fss Element (P: Number of test functions and $\mathrm{N}=$ Number of Basis functions).

\begin{tabular}{|l|c|r|c|c|c|}
\hline Used Method & Time for D1 & Time D2 & Conv. for D1 & Conv. for D2 & All Element \\
\hline MoM Method & 320 & 322 & $\mathrm{P}=16 \mathrm{~N}=3000$ & $\mathrm{P}=16 \mathrm{~N}=3000$ & $\left(\mathrm{Q}^{*} \mathrm{M}\right)^{*}(\mathrm{P}+\mathrm{N})$ \\
Hybrid Method & 15.65 & 15.75 & $\mathrm{P}=1 \mathrm{~N}=100$ & $\mathrm{P}=1 \mathrm{~N}=100$ & $\left(\mathrm{Q}^{*} \mathrm{M}\right)^{*}(\mathrm{P}+\mathrm{N})$ \\
\hline
\end{tabular}

Table2. Comparison of time consumed between the MoM and hybrid approach to computing the current distribution for A unit Fss Element (P: Number of test functions and $\mathrm{N}=$ Number of Basis functions)

\begin{tabular}{|l|l|r|l|l|l|}
\hline Used Method & Time for D1 & Time D2 & Conv. for D1 & Conv.for D2 & All Element \\
\hline MoM Method & 1320 & 1322 & $\mathrm{P}=16 \mathrm{~N}=3000$ & $\mathrm{P}=16 \mathrm{~N}=3000$ & $\left(\mathrm{Q}^{*} \mathrm{M}\right)^{*}(\mathrm{P}+\mathrm{N})$ \\
Hybrid Method & 25.65 & 25.51 & $\mathrm{P}=1 \mathrm{~N}=100$ & $\mathrm{P}=1 \mathrm{~N}=100$ & $\left(\mathrm{Q}^{*} \mathrm{M}\right)^{*}(\mathrm{P}+\mathrm{N})$ \\
\hline
\end{tabular}

- As we concluded from these 2 tables, for an Fss Structure of $(N * Q)$, time is multiplied by the fss's dimension .the hybrid approach indulges a considerable reduction and enhancement of the operation time, in addition, the number of modes to obtain convergence is considered reduced, these important advantages are guaranteed by the use of a single test function. proportionally, when the number of test function decreases, the size of the manipulated matrices decreases. the computational time will be reduced exponentially. 


\section{Conclusion}

In this paper, we present a new Hybrid method, that we lead a much less operation, and to reach the convergence quickly and faster than Mom Method because we use a single test function. our method combines the two asymptotic method the (PO) Optical physic and (GTD) General theory of Diffraction, the first applies to the surface of the antenna and gives us the distribution of the current, in this area to this function which models the shape of the current we add that determined by the GTD the second one, so we have an accuracy and complete function then we introduce the effect of the shaps by using the diffraction coefficient. consequently, the size of the operation manipulated in the matrices is enormously reduced. as the result of this, we reduce the numerical complexity and memory, CPU resources required for the problem.

\section{List of Abbreviations}

[MoM] Moment Method

[FSS] Frequency Selective Surface

[GTD] General Theory of Diffraction

[PO] Optical Physics

[GEC] General Equivalent Circuit

[FEM] Finite Element Method

[PEC] Perfect Electrical Conductor

\section{Declarations}

\section{1 availability of data and materials}

The authors declare that [the/all other] data supporting the findings of this study are available within the article [and its supplementary information files]

\subsection{Competing interests}

The authors declare that they have no competing interests.

\subsection{Funding}

Not Applicable

\subsection{Authors' contributions}

SM analyzed and interpreted the result data regarding the simulations, evaluation of the new hybrid Approch. TA performed the method, verified the results and its coherances. All authors read and approved the final manuscript. 


\subsection{Acknowledgements}

Not Applicable.

\section{Refereces}

[1] Tretyakov, S.A.; Glybovski, S.B.; Belov, P.A.; Kivshar, Y.S.; Simovski, C.R. Metasurfaces: From microwaves to visible. Phys. Rep. 2016, 634, 1-72.

[2] Munk, B.A. Frequency Selective Surfaces: Theory and Design; Wiley Online Library: Hoboken, NJ, USA, 2000; Volume 29.

[3] Costa, F.; Monorchio, A.; Manara, G. An overview of equivalent circuit modeling techniques of frequency selective surfaces and metasurfaces. Appl. Comput. Electromagn. Soc. J. 2014, 29, 960-976.

[4] Gianvittorio, J.P.; Romeu, J.; Blanch, S.; Rahmat-Samii, Y. Self-similar prefractal frequency selective surfaces for multiband and dual-polarized applications. IEEE Trans. Antennas Propag. 2003, 51, 3088-3096.

[5] Kern, D.J.; Werner, D.H.; Monorchio, A.; Lanuzza, L.; Wilhelm, M.J. The design synthesis of multiband artificial magnetic conductors using high impedance frequency selective surfaces. IEEE Trans. Antennas Propag. 2005, 53,8-17.

[6]. Yang, F.; Rahmat-Samii, Y. Electromagnetic Band Gap Structures in Antenna Engineering; Cambridge University Press: Cambridge, UK, 2009.

[7]. Kunz, K.S.; Luebbers, R.J. The Finite Difference Time Domain Method for Electromagnetics; CRC Press: Boca Raton, FL, USA, 1993.

[8]. Bagby, J.S.; Nyquist, D.P.; Drachman, B.C. Integral formulation for analysis of integrated dielectric waveguides. IEEE Trans. MTT-33 1985, 33, 906-915.

[9]. Koshiba, M.; Suzuki, M. Application of the Boundary-Element Method to Waveguide Discontinuities (Short Paper). IEEE Trans. Microw. Theory Tech. 1986, 34, 301-307.

[10]. Mink, J.W.; Schwering, F.K. Special Issue on Numerial Methods. IEEE Trans. Microw. Theory Tech. 1985,MTT-33, 10.

[11]. Chen, C.-C. Transmission through a conducting screen perforated periodically with apertures. IEEE Trans.Microw. Theory Tech. 1970, 18, 627-632.

[11]. Mittra, R.; Chan, C.H.; Cwik, T. Techniques for analyzing frequency selective surfaces-a review. Proc. IEEE 1988, 76, 1593-1615.

[12]. Orta, R.; Tascone, R.; Zich, R. A unified formulation for the analysis of general frequency selective surfaces.Electromagnetics 1985, 5, 307-329.

[13]. Bozzi, M.; Perregrini, L. Efficient analysis of thin conductive screens perforated periodically with arbitrarily shaped apertures. Electron. Lett. 1999, 35, 1085-1087.

[14] Costa, F.; Monorchio, A.; Manara, G. Efficient analysis of frequency-selective surfaces by a simple equivalent-circuit model. IEEE Antennas Propag. Mag. 2012, 54, $35-48$.

[15] Panwar, R.; Lee, J.R. Progress in frequency selective surface-based smart electromagnetic structures: A critical review. Aerosp. Sci. Technol. 2017, 66, 216-234. 
[CrossRef]

[16] Wang, J.; Qu, S.; Li, L.; Wang, J.; Feng, M.; Ma, H.; Du, H.; Xu, Z. All-dielectric metamaterial frequency selective surface. J. Adv. Dielectr. 2017, 7, 1730002. [CrossRef] [17] Mackay, A.; Sanz-Izquierdo, B.; Parker, E.A. Evolution of frequency selective surfaces. FERMAT 2014, 2, 1-7.

[18] Thakur, S.; Yadava, R.; Das, S. A review on Adaptive Frequency Selective Surfaces (AFSS) based patch antennas. In Proceedings of the Computing, Communications and IT Applications Conference (ComComAp),Hong Kong, China, 1-4 April 2013; pp. $120-124$. 
Figures

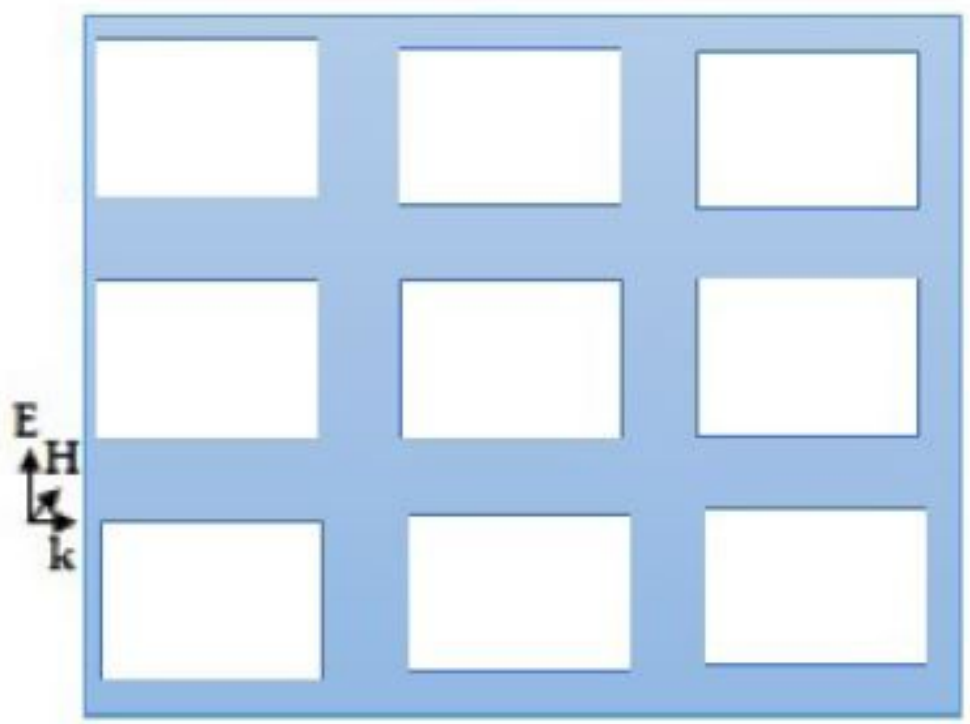

Figure 1

Surface waveguide made of metallic frames.

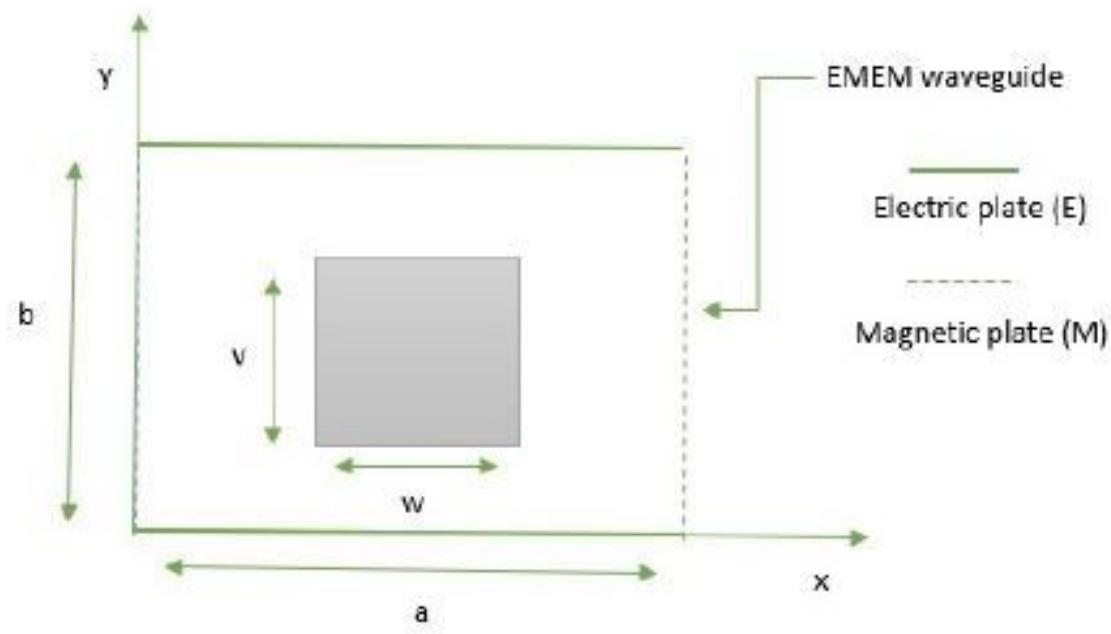

Figure 2

single Cell of FSS in waveguide. 


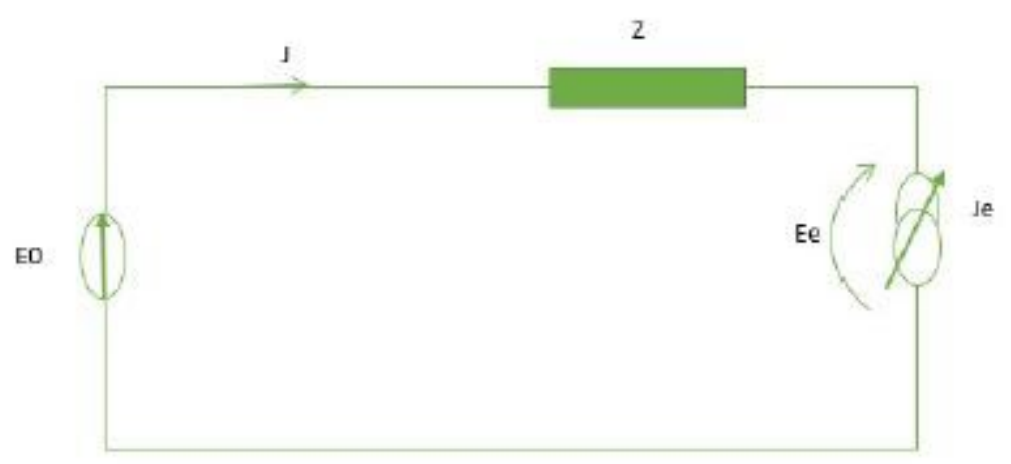

Figure 3

the Equivalent Circuit Method

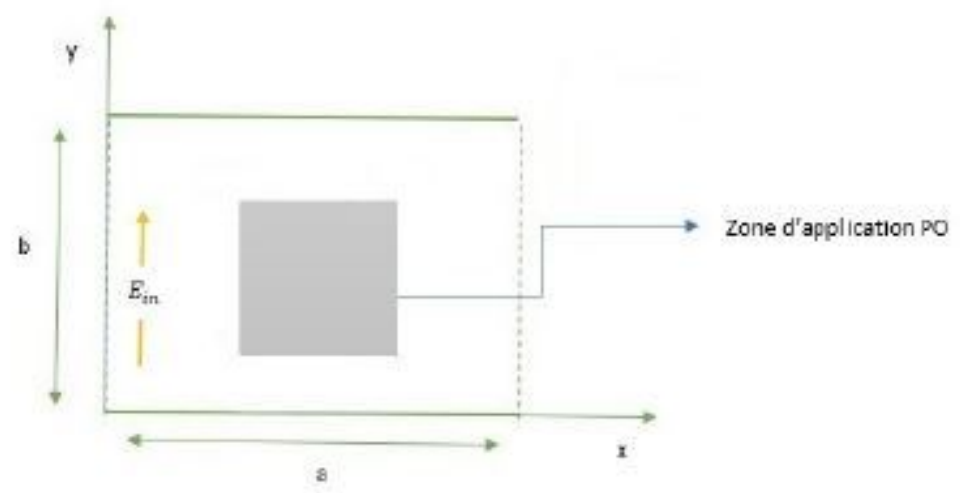

\section{Figure 4}

Mechanism of Optical Physics on the FSS unit

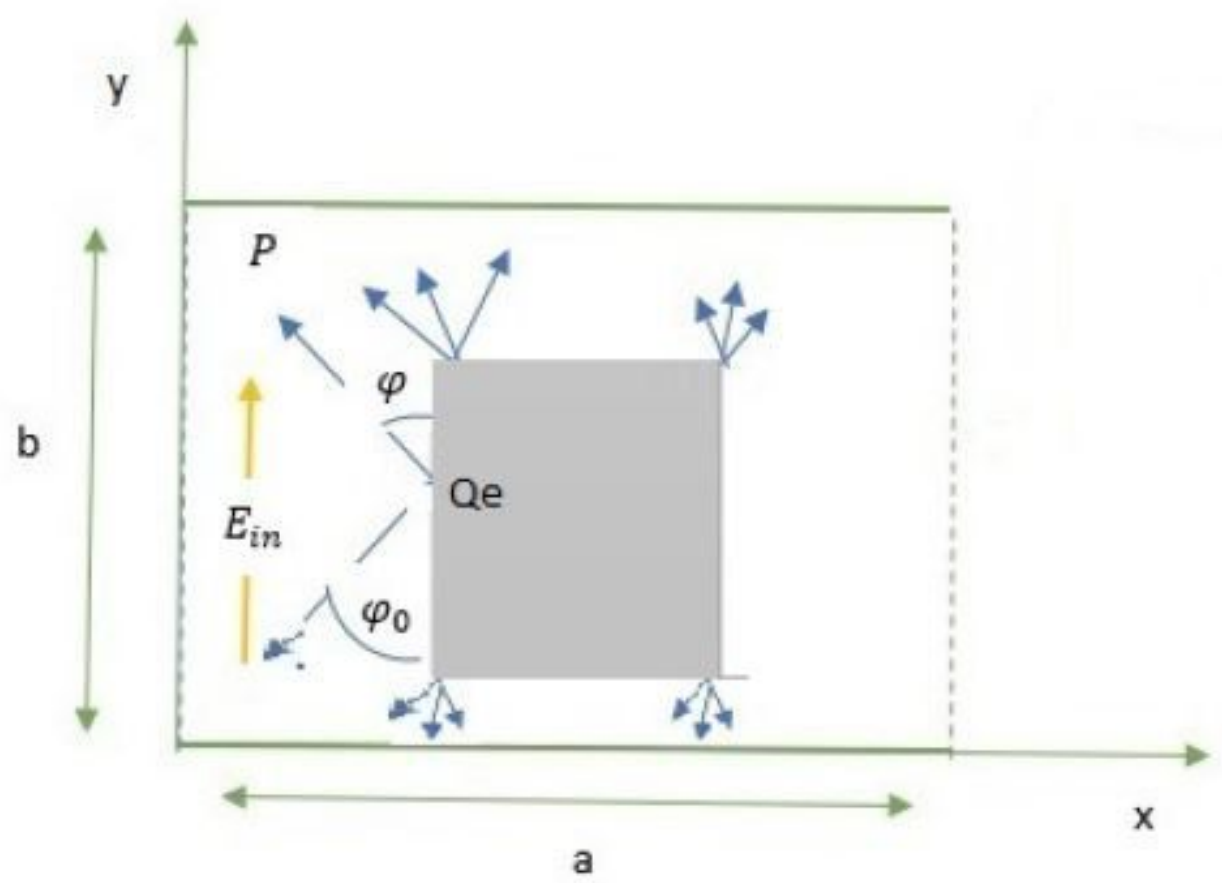


Figure 5

Diffraction of incident wave on the Edge and tips of the FSS unit

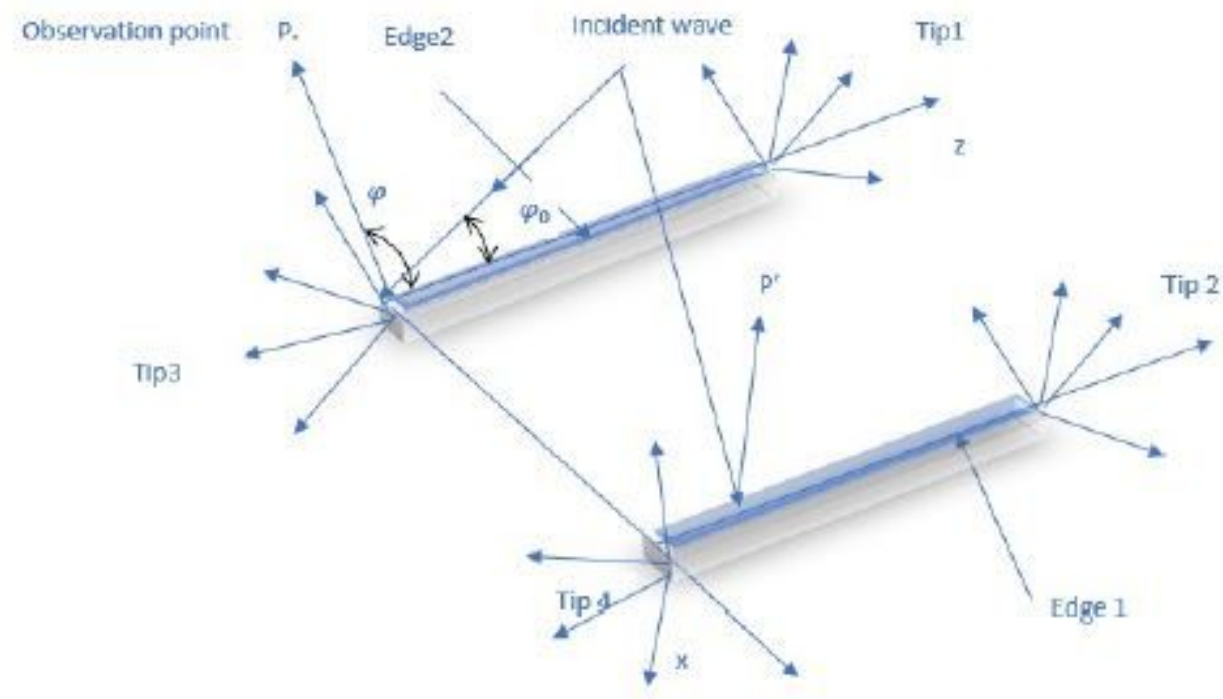

Figure 6

Modelization of FSS unit Edge and tips

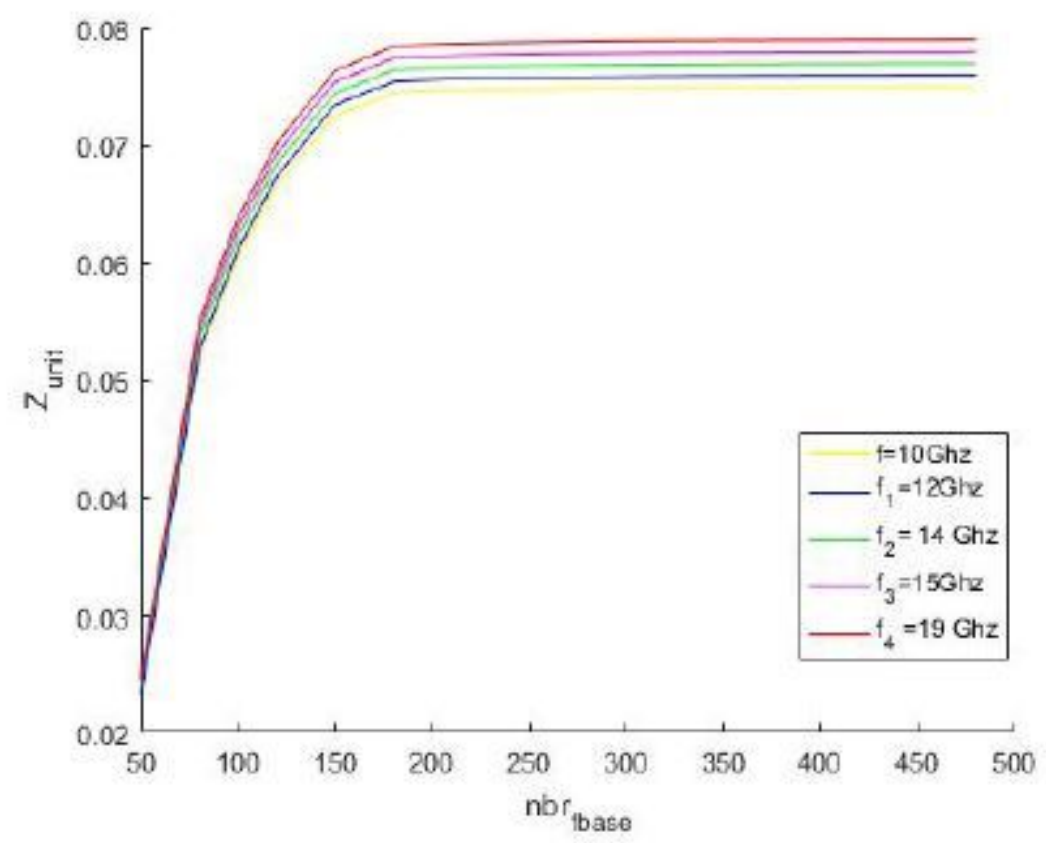

Figure 7

Input Impedance for different Number of Basics Function 


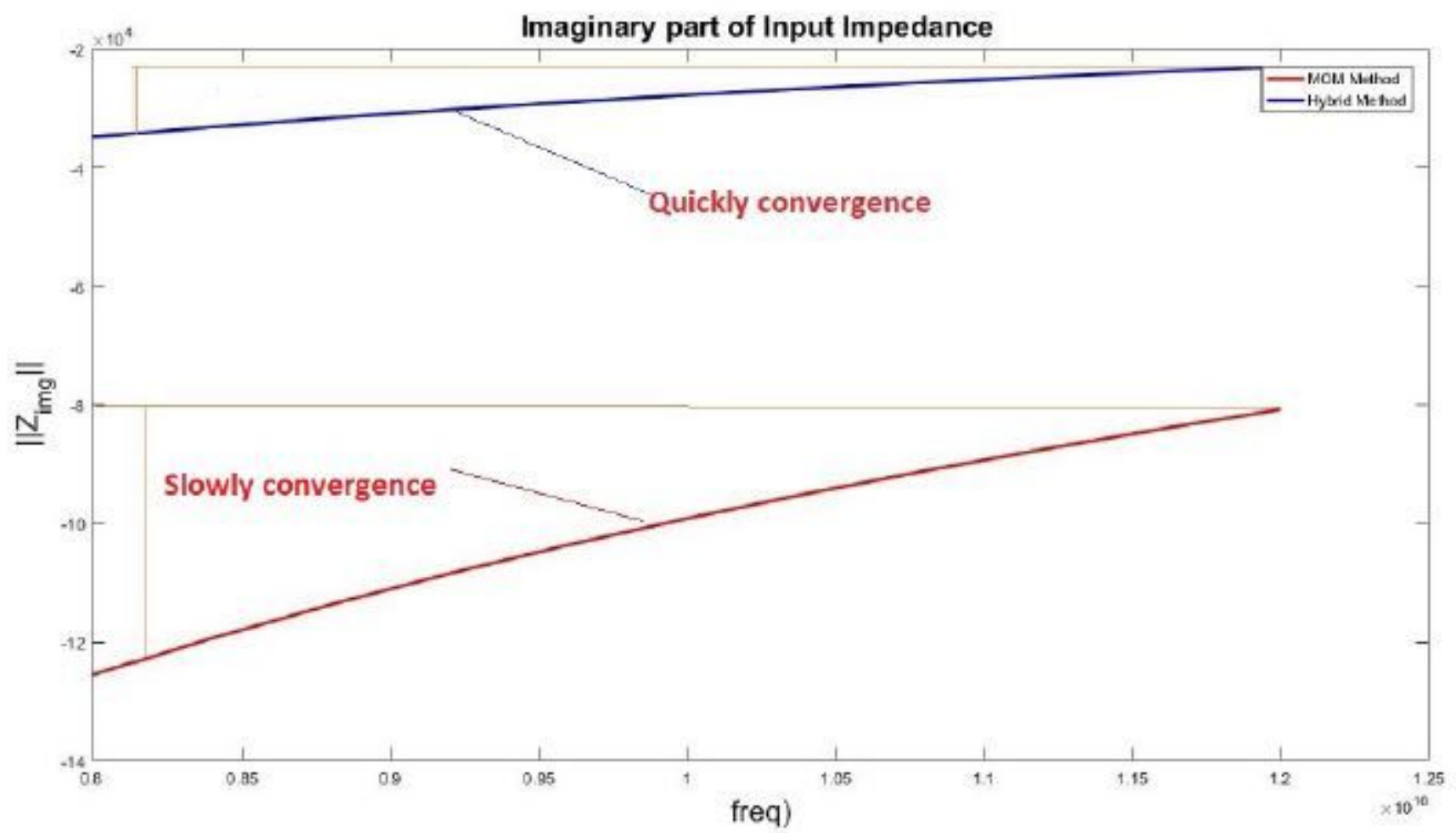

Figure 8

Comparaison between MoM Method and Hybrid Method of Imaginary Part of Input Impedance on the FSS Element

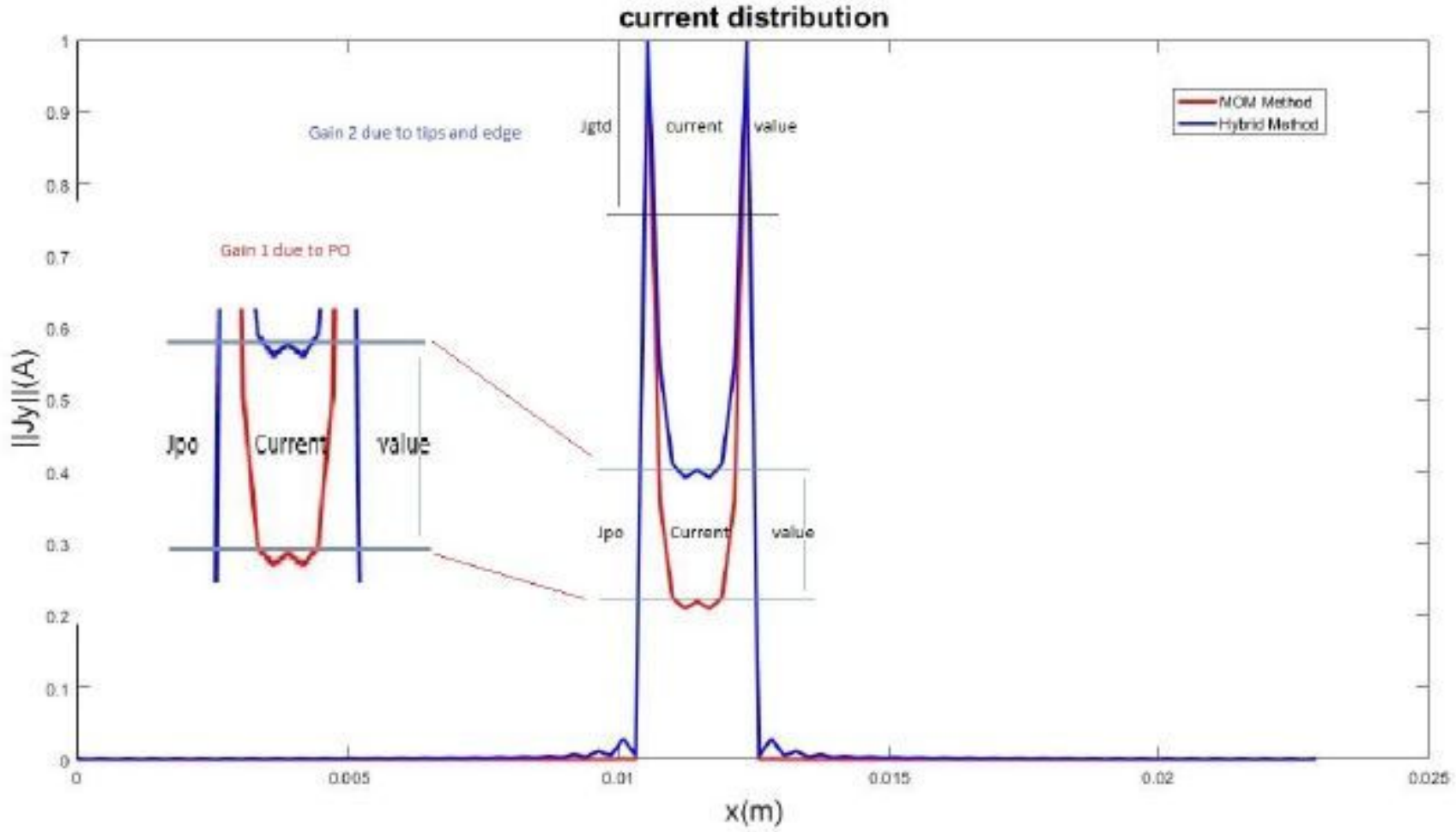


Figure 9

Comparaison between MoM Method and Hybrid Method of Current Distribution on the FSS Element

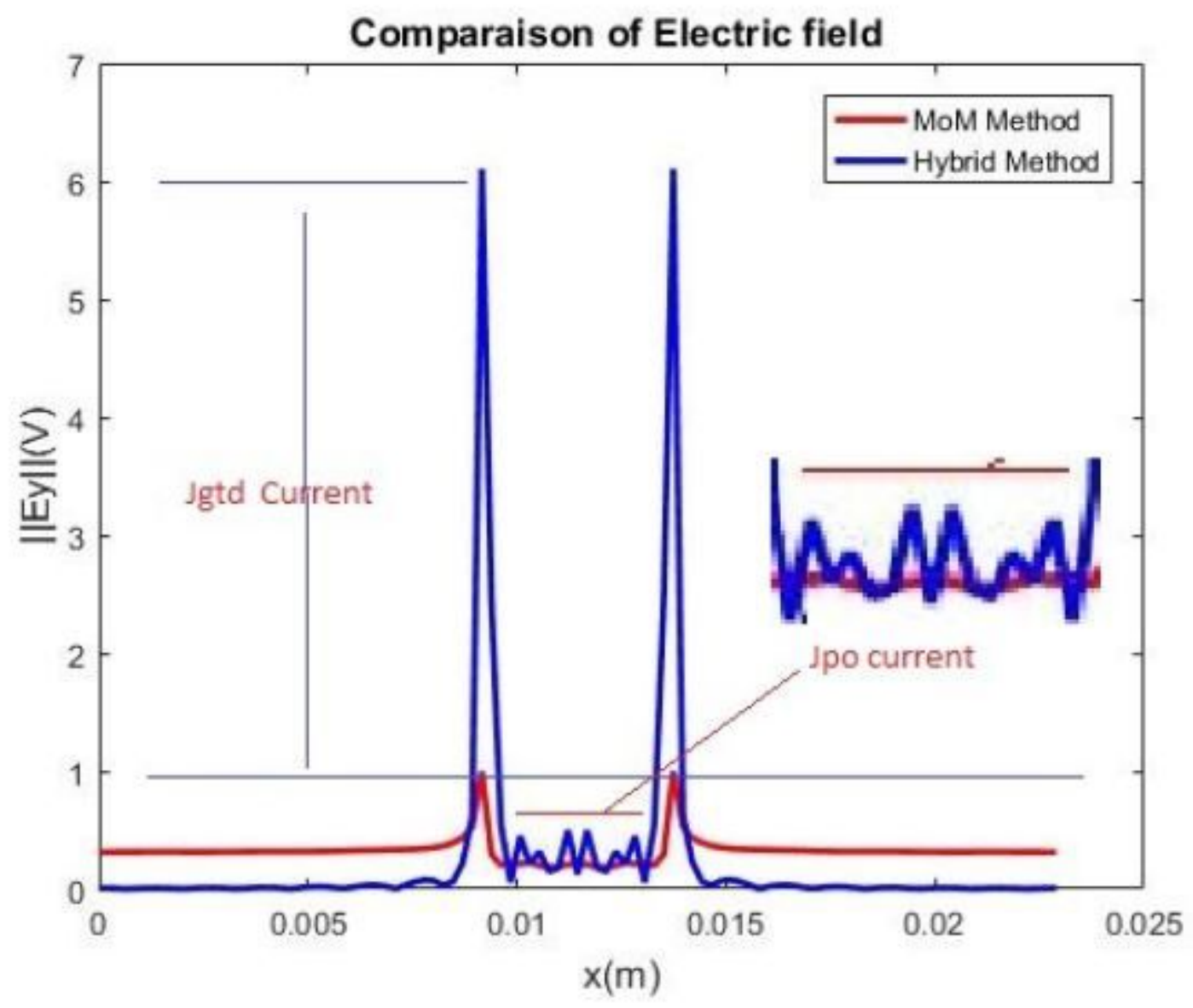

Figure 10

Comparaison between MoM Method and Hybrid Method of Electric Field on the FSS Element 


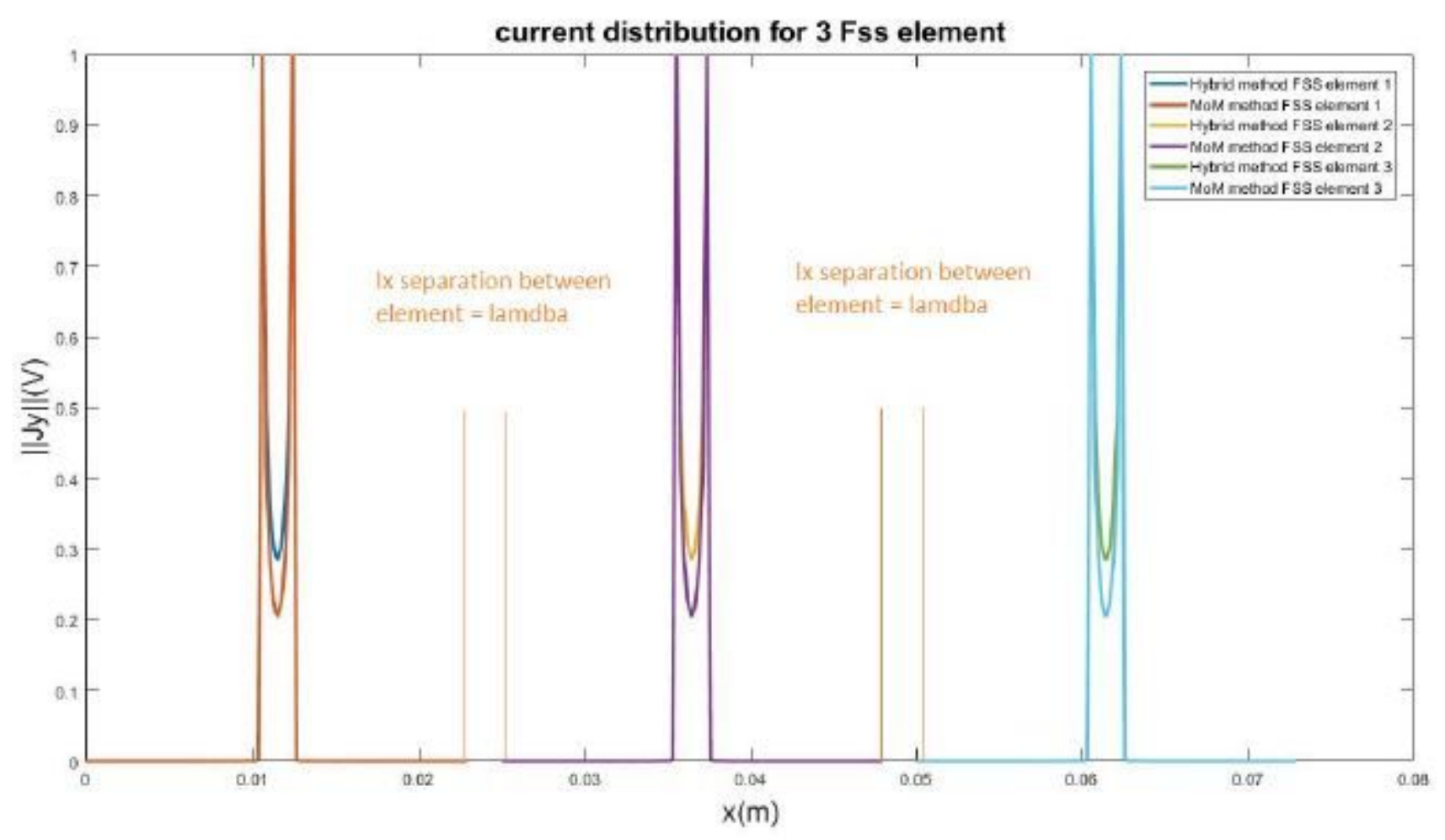

Figure 11

Current Distribution on the three FSS Element on X-axis

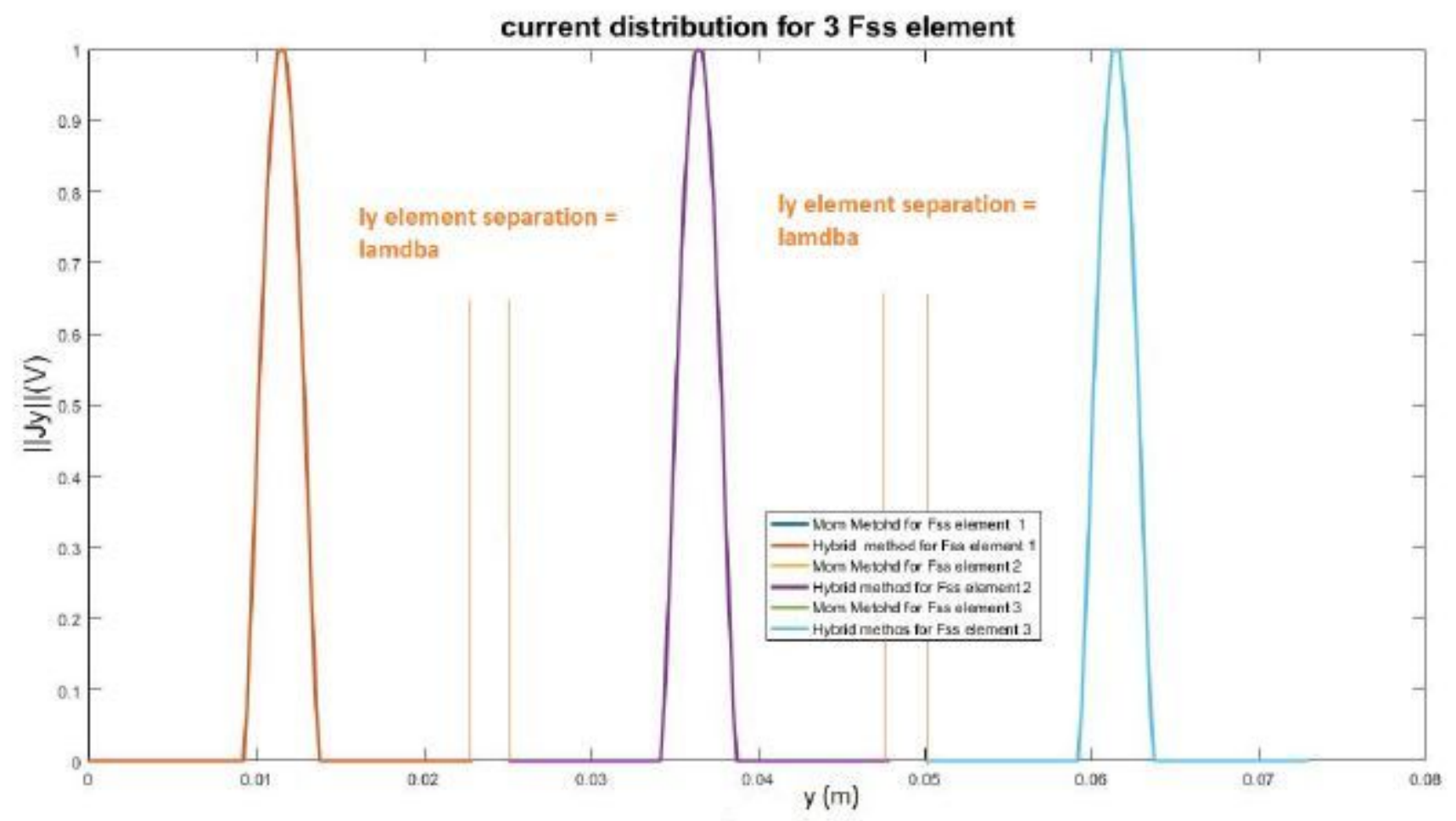

Figure 12 
Current Distribution on the three FSS Element on Y-axis 